\title{
NONAUTONOMOUS DIFFERENTIAL EQUATIONS AND TOPOLOGICAL DYNAMICS. II. LIMITING EQUATIONS
}

\author{
BY \\ GEORGE R. SELL( $\left.{ }^{1}\right)$
}

1. Introduction. In the preceding paper [22] we showed that the solutions of every admissible (nonautonomous) differential equation $x^{\prime}=f(x, t)$, defined on $W \times R$, can be viewed as a local dynamical system $\pi$ on $W \times \mathfrak{F}$, where $W$ is an open set in $R^{n}$ and $\mathfrak{F}$ is the space of translates of $f$. By introducing various topologies on $\mathfrak{F}$, we were able to study the topological-dynamical characteristics of the flow $\pi^{*}(f, t)=f_{t}$ on $\mathfrak{F}$. By introducing the concept of the "hull" of $f$ we were able to determine whether the flow $f_{t}$ be positively compact, compact, recurrent, almost periodic, etc.

In this paper we are primarily interested in the asymptotic behavior of the solutions of $x^{\prime}=f(x, t)$. In particular we shall investigate the relationship between the solution $\phi(x, f, t)$ of $x^{\prime}=f(x, t)$ and the corresponding motion $\pi(x, f ; t)$ in $W \times \mathfrak{F}$. One concept, which will play a central role in this investigation, is that of the "set of limiting equations," which is the $\omega$-limit set of the given differential equation $\left({ }^{2}\right)$. This study is carried out in $\$ \S 2$ and 3.

The concept of "asymptotically autonomous differential equations" introduced by L. Markus [8] can be described as those differential equations for which the $\omega$-limit set consists of a single point. This immediately admits generalizations to asymptotically-periodic, asymptotically-almost periodic and asymptoticallyrecurrent differential equations. One is able to derive more detailed information about the solutions of these equations.

In $\$ 4$ we shall investigate the question of the existence of almost periodic solutions and periodic solutions of differential equations. This study is related to the work of L. G. Deysach and G. R. Sell [4], J. L. Massera [9], R. K. Miller [12], C. R. Putnam [17], G. Seifert [19], and G. R. Sell [21].

In $\S 5$ we shall briefly discuss some extensions of our work.

Since this paper relies heavily on the results of [22], we shall presume knowledge of the earlier paper. We shall use the definitions and the notation of the preceding paper.

Received by the editors November 11, 1965.

(1) This research was supported in part by NSF Grant No. GP-3904.

$\left(^{2}\right)$ Whenever we attribute a dynamical, or topological-dynamical, property to a differential equation $x^{\prime}=f(x, t)$, this is to be interpreted as a property of the flow $f_{t}$ in the space of translates $\mathfrak{F}$, or in the hull $\mathfrak{F}^{*}$. 
In this paper we shall consider (almost exclusively) differential equations $x^{\prime}=f(x, t)$, where $f$ is a regular function in $\mathbb{}\left(W \times R, R^{n}\right)$, that is, every differential equation in the hull satisfies a uniqueness condition. Our modus operandi in $\S 2,3$, and 4 will be to treat first the situation with the compact-open topology on $\mathfrak{F}$. Generalizations of the results to other topologies on $\mathscr{F}$ will be discussed at the end of each section.

2. The solution $\phi(x, f, t)$ and the motion $\pi(x, f ; t)$. In this section we wish to examine the correspondence between solutions $\phi(x, f, t)$ of $x^{\prime}=f(x, t)$ and the motions $\pi(x, f ; t)$ in $W \times \mathfrak{F}$. We shall be primarily concerned here with the compactopen topology on $\mathfrak{F}$, and, unless stated to the contrary, the topological properties of the motions $f_{t}$ will be in terms of this topology.

A. Compact solutions. Let $f \in \mathfrak{C}\left(W \times R, R^{n}\right)$ be an admissible function and let $\phi(t)=\phi(x, f, t)$ be the solution of

$$
x^{\prime}=f(x, t)
$$

that satisfies $\phi(x, f, 0)=x$. Let $I_{x}=\left(\alpha_{x}, \beta_{x}\right)$ be the maximal interval of definition for $\phi$. We shall say that the solution $\phi$ is positively compact if the set $\left\{\phi(t): 0 \leqq t<\beta_{x}\right\}$ lies in a compact set in $W$. (Negative compactness and compactness are defined similarly.) Since $I_{x}$ is maximal, it follows that $\beta_{x}=+\infty$, whenever $\phi$ is positively compact. If $\phi$ is compact, then $I_{x}=R$, that is, $\phi$ is defined for all $t$ in $R$.

Let $f \in \mathfrak{C}\left(W \times R, R^{n}\right)$ be a regular function. Then the mapping (see Theorem 8 in [22])

$$
\pi(x, f ; t)=\left(\phi(x, f, t), f_{t}\right)
$$

defines a local dynamical system on $X=W \times \mathfrak{F}_{c o}^{*}$, where $\mathfrak{F}_{\mathrm{co}}^{*}$ is the hull of $f$. The next result, which establishes the basic relationship between the solutions $\phi(x, f, t)$ and the corresponding motions $\pi(x, f ; t)$, is now easily verified.

LemMa 1. Let $f \in \mathfrak{G}\left(W \times R, R^{n}\right)$ be a regular function.

A. If a motion $\pi(x, f ; t)$ in $W \times \mathfrak{F}_{c o}^{*}$ is

$$
\begin{gathered}
\left\{\begin{array}{l}
\text { positively compact } \\
\text { compact } \\
\text { periodic } \\
\text { almost periodic }
\end{array}\right\} \text {, then the motion } f_{t} \text { in } \mathfrak{F}_{\mathrm{c}}^{*} \text { is } \\
\left\{\begin{array}{l}
\text { positively compact } \\
\text { compact } \\
\text { periodic } \\
\text { almost periodic }
\end{array}\right\} \text { and the corresponding solution } \\
\phi(x, f, t) \text { is }\left\{\begin{array}{l}
\text { positively compact } \\
\text { compact } \\
\text { periodic } \\
\text { Bohr almost periodic }
\end{array}\right\}
\end{gathered}
$$


B. Assume that the motion $f_{t}$ in $\mathfrak{F}_{c o}^{*}$ is

$$
\begin{aligned}
& \left\{\begin{array}{l}
\text { positively compact } \\
\text { compact } \\
\text { almost periodic }
\end{array}\right\} . \text { Then a solution } \phi(x, f, t) \text { is } \\
& \left\{\begin{array}{l}
\text { positively compact } \\
\text { compact } \\
\text { Bohr almost periodic }
\end{array}\right\} \text { if and only if the corresponding motion } \\
& \pi(x, f ; t) \text { is }\left\{\begin{array}{l}
\text { positively compact } \\
\text { compact } \\
\text { almost periodic }
\end{array}\right\} .
\end{aligned}
$$

B. The positive limit set. Let $f \in \mathfrak{C}\left(W \times R, R^{n}\right)$ be a regular function and let $\pi$ be the local dynamical system on $W \times \mathfrak{F}_{\text {co }}^{*}$ given by (2). Then, the projections

$$
P: W \times \mathfrak{F}_{c o}^{*} \rightarrow W \text { and } Q: W \times \mathfrak{F}_{c o}^{*} \rightarrow \mathfrak{F}_{c o}^{*}
$$

are continuous. The motion $\pi(x, f ; t)$ is defined for all $t \geqq 0$ if and only if the solution $\phi(x, f, t)$ is defined for all $t \geqq 0$. In this case, the $\omega$-limit set $\Omega_{(x, f)}$ of the motion is defined, although it may be empty. We define the positive limit set of the solution $\phi(x, f, t)$ as

$$
L_{(x, f)}^{+}=P\left(\Omega_{(x, f)}\right) .
$$

If the motion $f_{t}$ in $\mathfrak{F}_{c o}^{*}$ is positively compact, then the positive limit set $L^{+}$can be characterized as follows:

LEMMA 2. Let $f \in \mathfrak{C}\left(W \times R, R^{n}\right)$ be a regular function and assume that the motion $f_{t}$ is positively compact. Then a point $\hat{x}$ lies in the positive limit set $L_{(x, f)}^{+}$ of the solution $\phi(x, f, t)$ if and only if there is a sequence $\left\{\tau_{n}\right\}$ in $R$ with

$$
\tau_{n} \rightarrow \infty \text { and } \phi\left(x, f, \tau_{n}\right) \rightarrow \hat{x} .
$$

Proof. Recall that a point $(\hat{x}, \hat{f})$ lies in the $\omega$-limit set $\Omega_{(x, f)}$ of the motion $\pi(x, f ; t)$ if and only if there is a sequence $\left\{\tau_{n}\right\}$ in $R$ with

$$
\tau_{n} \rightarrow \infty \text { and } \pi\left(x, f, \tau_{n}\right) \rightarrow(\hat{x}, \hat{f}) .
$$

The last limit can be rewritten as

$$
\phi\left(x, f, \tau_{n}\right) \rightarrow \hat{x} \text { and } f_{\tau_{n}} \rightarrow \hat{f} .
$$

Now assume that $\hat{x} \in L_{(x, f)}^{+}$. Then there is an $\hat{f}$ in $\mathfrak{F}_{c o}^{*}$ such that $(\hat{x}, \hat{f}) \in \Omega_{(x, f)}$. Hence there is a sequence $\left\{\tau_{n}\right\}$ in $R$ satisfying (3). Conversely, let $\hat{x} \in W$ and let $\left\{\tau_{n}\right\}$ be a sequence in $R$ that satisfies (3). Since the motion $f_{t}$ is positively compact, we can find a convergent subsequence of $\left\{f_{\tau_{n}}\right\}$, say that $f_{\tau_{n}} \rightarrow \hat{f}$. Hence, (4) is satisfied. It then follows that $(\hat{x}, \hat{f}) \in \Omega_{(x, f)}$, or that $\hat{x} \in L_{(x, f)}^{+}$. Q.E.D.

REMARK. The concept of the positive limit set seems to have been introduced first by L. Markus [8]. It has also been used by J. P. LaSalle [7] and R. K. Miller [11]. 
THEOREM 1. Let $f \in \mathfrak{S}\left(W \times R, R^{n}\right)$ be a regular function and let $\mathfrak{F}_{\mathrm{co}}^{*}$ be the hull of $f$ in the compact-open topology. Assume that $f_{t}$ is positively compact in the compactopen topology. Let $\phi(x, f, t)$ be a positively compact solution of $x^{\prime}=f(x, t)$. Then the w-limit set $\Omega_{(x, f)}$ in $W \times \mathfrak{F}_{\mathrm{co}}^{*}$ is nonempty, compact and invariant. If $\left(x^{*}, f^{*}\right) \in \Omega_{(x, f)}$, then the solution $\phi\left(x^{*}, f^{*}, t\right)$ of $x^{\prime}=f^{*}(x, t)$ is compact. Furthermore, the positive limit set $L_{(x, f)}^{+}$is a nonempty, compact set in $W$. Also, if $x^{*} \in L_{(x, f)}^{+}$, then $\phi\left(x^{*}, f^{*}, t\right) \in L_{(x, f)}^{+}$for all $t$, where $f^{*}$ is some function in $\mathfrak{F}_{\mathrm{co}}^{*}$.

Proof. By Lemma 1 , the motion $\pi(x, f, t)$ is positively compact in the local dynamical system on $X=W \times \mathfrak{F}_{\text {co. }}^{*}$. Therefore (by Lemma 3 of [22]) the $\omega$-limit set $\Omega_{(x, f)}$ is nonempty and compact. Since the projection $P: W \times \mathfrak{F}_{\mathrm{co}}^{*} \rightarrow W$ is continuous, the set $L_{(x, f)}^{+}=P\left(\Omega_{(x, f)}\right)$ is nonempty and compact. The rest of the theorem follows from Lemma 1. Q.E.D.

In this paper we are primarily interested in the topological-dynamical behavior of solutions of nonautonomous differential equations. However, in applications one may be interested in some analytical criteria for the dynamical properties. In particular, the reader will recall that the compactness, or positive-compactness, of the motion $f_{t}$ is implied by a uniform continuity condition on $f$. (See Theorem 14 and Corollaries in [22].) The following corollary of Theorem 1 will illustrate this.

Corollary. Let $f \in \mathfrak{S}\left(W \times R, R^{n}\right)$ be a regular function and let $\mathfrak{F}_{\mathrm{co}}^{*}$ be the hull of $f$. Assume that $f$ is bounded and uniformly continuous on every set $M \times R^{+}$, where $M$ is compact in $W$ and $R^{+}=\{t: 0 \leqq t<\infty\}$. If $\phi(x, f, t)$ is a positively compact solution of $x^{\prime}=f(x, t)$, then $\Omega_{(x, f)}$ is a nonempty, compact set in $W \times \mathfrak{F}_{c o}^{*}$ and $L_{(x, f)}^{+}$ is a nonempty, compact set in $W$.

Proof. This follows from the above theorem and Theorem 14 in [22]. Q.E.D.

A corollary similar to this can be stated after many theorems listed below. We shall not give a formal statement.

Since the mapping $\pi^{*}=Q \pi$, where $Q: W \times \mathfrak{F}_{\mathrm{co}}^{*} \rightarrow \mathfrak{F}_{\mathrm{co}}^{*}$ is the projection map, defines a dynamical system on $\mathfrak{F}_{\text {co }}^{*}$, it follows that if $\Omega_{(x, f)}$ is invariant in $W \times \mathfrak{F}_{\text {co }}^{*}$, then the set $Q\left(\Omega_{(x, f)}\right)$ in $\mathfrak{F}_{\text {co }}^{*}$ is an invariant set. It is, in fact, the $\omega$-limit set of the motion $f_{t}$ in $\mathfrak{F}_{\text {co }}^{*}$, that is, $\Omega_{f}^{*}=Q\left(\Omega_{(x, f)}\right)$. This set $\Omega_{f}^{*}$ is very important in the study of asymptotic behavior of solutions of (1). We shall pursue this study by a detailed analysis of the relationship between the solutions of (1) and the solutions of differential equations arising from $\Omega_{f}^{*}$.

REMARK. Let us note that if $f \in \mathfrak{C}\left(W \times R, R^{n}\right)$ is admissible and $\mathfrak{F}^{*}$ is any hull of $f$, then the above results can be generalized to this case, in the obvious manner. It should be noted that, in this case, the positive limit set $L_{(x, f)}^{+}$will depend on the hull $\mathfrak{F}^{*}$.

3. Limiting equations. In this section we shall consider regular functions $f$ in $\mathfrak{E}\left(W \times R, R^{n}\right)$. We shall consider first the case where the space of translates $\mathfrak{F}$, 
or the hull $\mathfrak{F}_{\mathrm{co}}^{*}$, has the compact-open topology. The generalizations of these results to other topologies will be treated at the end of this section.

A. Definition of limiting equations. Let $f \in \mathfrak{S}\left(W \times R, R^{n}\right)$ and let $\mathfrak{F}_{\mathrm{co}}^{*}$ be the hull of $f$. (Neither regularity nor admissibility of $f$ will be important here.) Let $\pi^{*}(f, t)=f_{t}$ be the flow on $\mathfrak{F}_{\mathrm{co}}^{*}$ and let $\Omega_{f}^{*}$ denote the $\omega$-limit set of $f$ in this flow. If the $\omega$-limit set $\Omega_{f}^{*}$ of $f$ in $\mathfrak{F}_{\mathrm{co}}^{*}$ is nonempty, then we say that the set of limiting equations for

$$
x^{\prime}=f(x, t)
$$

is the set of all differential equations of the form

$$
x^{\prime}=f^{*}(x, t) \quad\left(f^{*} \in \Omega_{f}^{*}\right) .
$$

The first result is elementary.

Lemma 3. Let $f \in \mathbb{C}\left(W \times R, R^{n}\right)$ and assume that the motion $f_{t}$ is positively compact in the compact-open topology. Then the set of limit equations for $f$ is a nonempty, compact subset of $\mathfrak{F}_{\mathrm{co}}^{*} \subset \mathfrak{\subseteq}\left(W \times R, R^{n}\right)$.

Let $f \in \mathfrak{C}\left(W \times R, R^{n}\right)$ and assume that the motion $f_{t}$ is positively compact in the compact-open topology. Let $\Omega_{f}^{*}$ be the $\omega$-limit set of $f$ in $\mathfrak{F}_{\text {co }}^{*}$. We shall say that $f$ is asymptotically autonomous if $\Omega_{f}^{*}$ consists of a simple point, say $\Omega_{f}^{*}=\left\{f^{*}\right\}$. (This means (see Theorem 13 in [22]) that $f^{*}$ is an autonomous function.) We shall say that $f$ is asymptotically periodic if $\Omega_{f}^{*}$ consists of a single periodic trajectory. (This means (see Theorem 13 in [22]) that if $f^{*} \in \Omega_{f}^{*}$, then $f^{*}$ is periodic in $t$.) We shall say that $f$ is asymptotically almost periodic if $\Omega_{f}^{*}$ is an a.p. minimal set in $\mathfrak{F}_{\mathrm{co}}^{*}$. (This means (see Theorem 18 in [22]) that if $f^{*} \in \Omega_{f}^{*}$, then $f^{*}$ is a Bohr almost periodic function.) We shall say that $f$ is asymptotically recurrent if $\Omega_{f}^{*}$ is a (compact) minimal set in $\mathfrak{F}_{\text {co }}^{*}$. Also, $f$ is said to be asymptotically Poisson-stable if there is a point $f^{*}$ in $\Omega_{f}^{*}$ such that the trajectory $f_{t}^{*}$ is dense in $\Omega_{f}^{*}$. (A necessary and sufficient condition that $\Omega_{f}^{*}$ have this property is that it be "quasi-minimal." See V. V. Nemyckiĭ [15] or V. V. Nemyckiĭ and V. V. Stepanov [14] for a proof. This, of course, implies that the motion $f_{t}^{*}$ is Poisson-stable.)

If $f$ is asymptotically autonomous, then the set of limit equations for $x^{\prime}=f(x, t)$ consists of the single equation $x^{\prime}=f^{*}(x)$, where $\Omega_{f}^{*}=\left\{f^{*}\right\}$. If $f$ is asymptotically periodic, then the set of limiting equations for $x^{\prime}=f(x, t)$ is given by

$$
x^{\prime}=f^{*}(x, \tau+t), \quad 0 \leqq \tau<P,
$$

where $f^{*} \in \Omega_{f}^{*}$ and $f^{*}$ is periodic in $t$ with (minimal) period $P$. The set of limiting equations in any of the other special cases can be constructed in a similar manner.

The following lemma will be helpful in some applications:

LEMMA 4. Let $f \in \mathfrak{C}\left(W \times R, R^{n}\right)$ and assume that $f=g+h$, where $g$ and $h$ are in $\mathfrak{S}\left(W \times R, R^{n}\right)$. Assume further that $h(x, t) \rightarrow 0$ as $t \rightarrow \infty$, uniformly on compact sets in $W$. The following statements are valid in the compact-open topology. 
(A) The limiting equations of $x^{\prime}=f(x, t)$ and $x^{\prime}=g(x, t)$ are the same.

(B) If $g_{t}$ is a compact motion, then $f_{t}$ is positively compact.

(C) If $g$ is Bohr almost periodic, then $f_{t}$ is positively compact.

(D) If $g$ is, respectively, autonomous, periodic in $t$, or Bohr almost periodic, then $f$ is, respectively, asymptotically autonomous, asymptotically periodic, or asymptotically almost periodic.

B. Solutions of the limiting equations. Now consider $f \in \mathbb{E}\left(W \times R, R^{n}\right)$ to be a regular function. The next result relates the behavior of a positively compact solution $\phi(x, f, t)$ of $x^{\prime}=f(x, t)$ with the behavior of solutions of the limiting equations.

THEOREM 2. Let $f \in \mathbb{S}\left(W \times R, R^{n}\right)$ be a regular function and assume that the motion $f_{t}$ is positively compact in $\mathfrak{F}_{\mathrm{co}}^{*}$. Let $\phi(x, f, t)$ be a positively compact solution of $x^{\prime}=f(x, t)$. Then for every point $\left(x^{*}, f^{*}\right)$ in $\Omega_{(x, f)}$, the solution $\phi\left(x^{*}, f^{*}, t\right)$ is compact. Moreover, there exists a sequence $\left\{\tau_{n}\right\}$ in $R$ with $\tau_{n} \rightarrow \infty$ and such that $\phi\left(x, f, \tau_{n}+t\right)$ converges to $\phi\left(x^{*}, f^{*}, t\right)$ uniformly on compact set in $R$.

Proof. This theorem is a consequence of the following fact from topological dynamics: If $\pi$ is a local dynamical system on $X$ and $\left\{p_{n}\right\}$ is a sequence that converges to $p$, then the sequence $\left\{\pi\left(p_{n}, t\right)\right\}$ converges to $\pi(p, t)$ uniformly on compact sets in $I_{p}$, where $I_{p}$ is the maximal interval of definition of $\pi(p, t)$. (See Lemma 4 of [22].)

Let $\phi(x, f, t)$ be a positively compact solution of $x^{\prime}=f(x, t)$. Then by Lemma 1 , the motion $\pi(x, f ; t)$ in the local dynamical system on $W \times \mathfrak{F}_{\text {co }}^{*}$ is positively compact. By Theorem 1, every solution $\phi\left(x^{*}, f^{*}, t\right)$, where $\left(x^{*}, f^{*}\right) \in \Omega_{(x, f)}$, is compact. Therefore, (see Lemma 3 of [22]) the solution $\phi\left(x^{*}, f^{*}, t\right)$ and the motion $\pi\left(x^{*}, f^{*}, t\right)$ are defined on all of $R$, that is, $I_{\left(x^{*}, f^{*}\right)}=R$. Now let $\left\{\tau_{n}\right\}$ be a sequence in $R$, with $\tau_{n} \rightarrow \infty$ and $\pi\left(x, f ; \tau_{n}\right) \rightarrow\left(x^{*}, f^{*}\right)$. Then, as cited above, $\pi\left(x, f ; \tau_{n}+t\right)$ converges to $\pi\left(x^{*}, f^{*} ; t\right)$ uniformly on compact sets in $R$. Since the projection $P$ is continuous, the solutions

$$
\phi\left(x, f, \tau_{n}+t\right)=P \pi\left(x, f, \tau_{n}+t\right)
$$

converge to $\phi\left(x^{*}, f^{*}, t\right)$ uniformly on compact sets in $R$. Q.E.D.

The conclusion of Theorem 2 can be formulated in terms of the positive limit set $L_{(x, f)}^{+}$. The basic fact here is that if $x^{*} \in L_{(x, f)}^{+}$, then there is an $f^{*}$ in $\mathfrak{F}_{c o}^{*}$ such that $\left(x^{*}, f^{*}\right) \in \Omega_{(x, f)}$.

COROllary 1. Let $f \in \mathbb{C}\left(W \times R, R^{n}\right)$ be a regular function and assume that the motion $f_{t}$ is positively compact in $\mathfrak{F}_{\mathrm{co}}^{*}$. Let $\phi(x, f, t)$ be a positively compact solution of $x^{\prime}=f(x, t)$. Then for every point $x^{*}$ in $L_{(x, f)}^{+}$, there is a function $f^{*}$ in $\Omega_{f}^{*}$ such that the solution $\phi\left(x^{*}, f^{*}, t\right)$ is compact. Moreover, there exists a sequence $\left\{\tau_{n}\right\}$ with $\tau_{n} \rightarrow \infty$ and such that $\phi\left(x, f, \tau_{n}+t\right)$ converges to $\phi\left(x^{*}, f^{*}, t\right)$ uniformly on compact sets in $R$. 
This corollary asserts that the positive limit set $L_{(x, f)}^{+}$is quasi-invariant in the sense defined by R. K. Miller [11].

If $f$ is asymptotically autonomous, then one can say more.

COROLlaRy 2. Let $f \in \mathfrak{C}\left(W \times R, R^{n}\right)$ be a regular function that is asymptotically autonomous. Let $\Omega_{f}^{*}=\left\{f^{*}\right\}$. Then every $\omega$-limit set $\Omega_{(x, f)}$ can be expressed in the form

$$
\Omega_{(x, f)}=L_{(x, f)}^{+} \times\left\{f^{*}\right\} .
$$

Therefore, the positive limit set $L_{(x, f)}^{+}$is the union of solutions of $x^{\prime}=f^{*}(x)$. If the solution $\phi(x, f, t)$ is positively compact, then for every $x^{*}$ in $L_{(x, f)}^{+}$the solution $\phi\left(x^{*}, f^{*}, t\right)$ is compact, and there exists a sequence $\left\{\tau_{n}\right\}$, with $\tau_{n} \rightarrow \infty$, such that $\phi\left(x, f, \tau_{n}+t\right)$ converges to $\phi\left(x^{*}, f^{*}, t\right)$ uniformly on compact sets in $R$.

The situation for asymptotically periodic equations is similar to that for asymptotically autonomous equations.

COROllaRY 3. Let $f \in \mathfrak{E}\left(W \times R, R^{n}\right)$ be a regular function that is asymptotically periodic and let $f^{*}$ be a (fixed) element of $\Omega_{f}^{*}$. Let $P$ be the minimal period of $f^{*}$ and let $\phi(x, f, t)$ be a positively compact solution of $x^{\prime}=f(x, t)$. Then for every $x^{*}$ in $L_{(x, f)}^{+}$there is a $\tau, 0 \leqq \tau<P$, such that the solution $\phi\left(x^{*}, f_{\tau}^{*}, t\right)$ of $x^{\prime}=f^{*}(x, \tau+t)$ is compact. Moreover, there exists a sequence $\left\{\tau_{n}\right\}$, with $\tau_{n} \rightarrow \infty$, such that $\phi\left(x, f, \tau_{n}+t\right)$ converges to $\phi\left(x^{*}, f_{\tau}^{*}, t\right)$ uniformly on compact sets in $R$.

The next corollary is merely the contrapositive of Theorem 2.

COROLlaRY 4. Let $f \in \mathfrak{C}\left(W \times R, R^{n}\right)$ be a regular function and assume that the motion $f_{t}$ is positively compact in $\mathfrak{F}_{c o}^{*}$. If there is one limiting equation $x^{\prime}=f^{*}(x, t)$ that does not have a compact solution, then the given equation $x^{\prime}=f(x, t)$ does not have a positively compact motion.

REMARKS. The above results generalize some work of L. Markus [8] and R. K. Miller [11]. In Example A (below) we show that it is possible that the given equation may have no positively compact solutions, while the limiting equations have only compact solutions.

A form of Theorem 2 is possible even if we drop the assumption of positive compactness. We shall state the following theorem without proof.

TheOREM 3. Let $f \in \mathfrak{S}\left(W \times R, R^{n}\right)$ be a regular function and let $\phi(x, f, t)$ be a solution of $x^{\prime}=f(x, t)$ that is defined for all $t \geqq 0$. Let $\Omega_{(x, f)}$ be the w-limit set of the motion $\pi(x, f ; t)$ in $W \times \mathfrak{F}_{\mathrm{co}}^{*}$. If $\left(x^{*}, f^{*}\right) \in \Omega_{(x, f)}$, then there is a sequence $\left\{\tau_{n}\right\}$ with $\tau_{n} \rightarrow \infty$ and such that $\phi\left(x, f, \tau_{n}+t\right)$ converges to $\phi\left(x^{*}, f^{*}, t\right)$ uniformly on compact sets in $I_{x^{*}}$, where $I_{x^{*}}$ is the maximal interval of definition of the solution $\phi\left(x^{*}, f^{*}, t\right)$.

C. Stable solutions. We have already asserted (see Example A below) that the solutions of the given equation may be nonpositively compact while the solutions of the limiting equations are compact. This occurs when the given differential 
equation fails to have certain stability properties. Let us now investigate the asymptotic behavior of solutions under the assumption of stability.

Let $f \in \mathbb{S}\left(W \times R, R^{n}\right)$ be a regular function and assume that $f(0, t)=0$ for all $t \geqq 0$. That is, $\phi(0, f, t)=0$ for all $t \geqq 0$. It follows then that $f^{*}(0, t)=0$ (for all $t \geqq 0$ ) for every function $f^{*}$ in the hull $\mathfrak{F}_{\text {co }}^{*}$, and that $f^{*}(0, t)=0$ (for all $t$ ) when $f^{*} \in \Omega_{f}^{*}$.

We shall need the following lemma.

LEMMA 5. Let $\alpha(r, t)$ be a nonnegative function defined for

$$
\{(r, t): 0 \leqq r \leqq a, 0 \leqq t<\infty\}
$$

that is nondecreasing in $r$. Let $f \in \mathcal{C}\left(W \times R, R^{n}\right)$ be a regular function. If the solutions of $x^{\prime}=f_{\tau}(x, t)$ satisfy

$$
\left|\phi\left(x, f_{\tau}, t\right)\right| \leqq \alpha(|x|, t) \quad(|x| \leqq a, 0 \leqq \tau, 0 \leqq t),
$$

then the solutions of $x^{\prime}=f^{*}(x, t)$ satisfy

$$
\left|\phi\left(x, f^{*}, t\right)\right| \leqq \alpha(|x|, t) \quad(|x| \leqq a, 0 \leqq t)
$$

for every limiting equation $f^{*} \in \Omega_{f}^{*}$.

Proof. Let $\varepsilon>0$ be given and let $I$ be any compact interval in

$$
R^{+}=\{t: 0 \leqq t<\infty\}
$$

Let $\rho$ be any basic metric on $\Subset\left(W \times R, R^{n}\right)$. By Kamke's Lemma (Lemma 2 in [22]), for every $f^{*}$ in $\Omega_{f}^{*}$ there is a $\delta>0$ such that

$$
\left|\phi\left(x, f^{*}, t\right)-\phi(x, g, t)\right|<\varepsilon \quad(|x| \leqq a, t \in I),
$$

whenever $\rho\left(f^{*}, g\right)<\delta$. By the definition of $\Omega_{f}^{*}$ we can find a translate $f_{\tau}, \tau \geqq 0$, such that $\rho\left(f^{*}, f_{\tau}\right)<\delta$. Then by combining (4) and (6) we get

$$
\left|\phi\left(x, f^{*}, t\right)\right| \leqq \varepsilon+\alpha(|x|, t) \quad(|x| \leqq a, t \in I) .
$$

Now let $\varepsilon \rightarrow 0$ and we get

$$
\left|\phi\left(x, f^{*}, t\right)\right| \leqq \alpha(|x|, t) \quad(|x| \leqq a, t \in I) .
$$

Since the right side of (7) does not depend on $I$, the result is true for all $t \geqq 0$. Q.E.D.

REMARK. It should be noted that in the last result we did not assume the motion $f_{t}$ to be positively compact. If $f_{t}$ is not positively compact, then the $\omega$-limit set $\Omega_{f}^{*}$ may be empty, in which case the conclusion of Lemma 5 is vacuously true. Also note that Theorems 4 and 5 stated below do not require the motion to be positively compact.

We give the following definitions of stability. 
Definitions. Let $f \in \mathfrak{C}\left(W \times R, R^{n}\right)$ be an admissible function with $f(0, t)=0$ for all $t \geqq 0$. We shall say that the null solution $\phi(0, f, t)=0$ is:

(i) stable if

$$
|\phi(x, f, t)| \leqq \alpha(|x|) \quad(|x| \leqq a, 0 \leqq t)
$$

where $\alpha(r)$ is a nonnegative, continuous, increasing function defined for $0 \leqq r \leqq a$ with $\alpha(0)=0$;

(ii) uniformly stable if

$$
\left|\phi\left(x, f_{\tau}, t\right)\right| \leqq \alpha(|x|) \quad(|x| \leqq a, 0 \leqq \tau, 0 \leqq t),
$$

where $\alpha$ is as in (i);

(iii) asymptotically stable if it is stable and $\phi\left(x, f_{\tau}, t\right) \rightarrow 0$ as $t \rightarrow \infty$, whenever $|x| \leqq a$ and $0 \leqq \tau$;

(iv) uniformly asymptotically stable if

$$
\left|\phi\left(x, f_{\tau}, t\right)\right| \leqq \alpha(|x|) \sigma(t) \quad(|x| \leqq a, 0 \leqq \tau, 0 \leqq t),
$$

where $\alpha$ is as in (i) and $\sigma(t)$ is a positive, continuous, decreasing function defined for $0 \leqq t$ with $\sigma(t) \rightarrow 0$ as $t \rightarrow \infty$. (For a discussion of the equivalence between these definitions of stability and the " $\varepsilon-\delta$ " definitions, see W. Hahn [5].)

The next theorem is now an immediate consequence of Lemma 5 and the above definitions.

THEOREM 4. Let $f \in \mathbb{E}\left(W \times R, R^{n}\right)$ be a regular function with $f(0, t)=0$ for all $t \geqq 0$.

(A) If the null solution of $x^{\prime}=f(x, t)$ is uniformly stable, then the null solution of every limiting equation $x^{\prime}=f^{*}(x, t)$ is uniformly stable.

(B) If the null solution of $x^{\prime}=f(x, t)$ is uniformly asymptotically stable, then the null solution of every limiting equation $x^{\prime}=f^{*}(x, t)$ is uniformly asymptotically stable.

The concept of stability for an arbitrary solution can be reduced to the above concept by a standard technique. That is, if $\phi$ is any solution of $x^{\prime}=f(x, t)$, then $\phi$ is said to be "- stable" if the null solution of

$$
x^{\prime}=f(x+\phi(t), t)-f(\phi(t), t)
$$

is - stable. The last theorem can now be generalized as follows:

THEOREM 5. Let $f \in \mathfrak{C}\left(W \times R, R^{n}\right)$ be a regular function. If there exists a positively compact solution $\phi(x, f, t)$ of $x^{\prime}=f(x, t)$ that is uniformly stable \{or, uniformly asymptotically stable $\}$, then every limiting equation $x^{\prime}=f^{*}(x, t)$ has a compact solution that is uniformly stable $\{$ or, respectively, uniformly asymptotically stable $\}$.

In Theorems 4 and 5 we assumed that the given equation had a "stable" solution and showed that the limiting equations had the same property. The 
problem of reversing these roles is a bit delicate. That is, if we assume some stability properties of the solutions of the limiting equations, then it is generally harder to derive results about the given equation. Consider the following example.

EXAmple A. The solutions of the linear equation

$$
x^{\prime}=\frac{1}{(t+2) \log (t+2)} x \quad(t \geqq 0)
$$

are neither positively compact, nor stable. However, the solutions of the limiting equation (there is only one) $x^{\prime}=0$ are compact and uniformly stable. (Although the above equation is defined only for $t \geqq 0$, one can easily extend it for $t<0$. This would not change the limiting equation.)

However, we are able to prove the following result for asymptotic stability.

THEOREM 6. Let $f \in \mathbb{C}\left(W \times R, R^{n}\right)$ be a regular function with $f(0, t)=0(t \geqq 0)$ and assume that the motion $f_{t}$ is positively compact, in the compact open topology. If

(i) the null solution of $x^{\prime}=f(x, t)$ is uniformly stable, and

(ii) the null solution of every limiting equation is asymptotically stable (in a uniform sense), that is $\left|\phi\left(x, f^{*}, t\right)\right| \rightarrow 0$ as $t \rightarrow \infty$ whenever $|x| \leqq a$ and $f^{*} \in \Omega_{f}^{*}$, then the null solution of the given equation $x^{\prime}=f(x, t)$ is asymptotically stable.

Proof. Let $\alpha(r)$ be defined for $0 \leqq r \leqq a$ so that (8) is satisfied. Then by Lemma 5 one has

$$
\left|\phi\left(x, f^{*}, t\right)\right| \leqq \alpha(|x|) \quad(|x| \leqq a, 0 \leqq t),
$$

for every $f^{*}$ in $\Omega_{f}^{*}$. Now consider the restriction of the local dynamical system on $W \times \mathfrak{F}_{\text {co }}^{*}$ to the subspace $W \times \Omega_{f}^{*}$. Let $M$ be the set $\{0\} \times \Omega_{f}^{*}$. In the sense of $\mathrm{J}$. Auslander, et al. [2], the set $M$ is a stable attractor in the space $W \times \Omega_{f}^{*}$. (In [2], the concept of an attractor was defined for dynamical systems. However, the definitions, and the results we need here, are valid for local dynamical systems.) The region of attraction $A(M)$ is an open, positively-invariant set in $W \times \Omega_{f}^{*}$ and it contains a set of the form $\left\{\left(x, f^{*}\right):|x| \leqq \hat{a}, f^{*} \in \Omega_{f}^{*}\right\}$, for some $\hat{a}>0$.

Now consider a solution $\phi\left(x, f_{\tau}, t\right)$ of $x^{\prime}=f_{\tau}(x, t)$, where $|x|$ is sufficiently small; that is,

$$
|x| \leqq a \text { and } \alpha(|x|) \leqq \hat{a} .
$$

Since $|x| \leqq a$, the solution $\phi\left(x, f_{\tau}, t\right)$ is positively compact and the $\omega$-limit set $\Omega=\Omega_{\left(x, f_{z}\right)}$ is nonempty and compact, and is contained in $W \times \Omega_{f}^{*}$. Also, since $\left|\phi\left(x, f_{\imath}, t\right)\right| \leqq \alpha(|x|) \leqq \hat{a}$, the $\omega$-limit set $\Omega$ lies in the region of attraction $A(M)$ of $M$. Since $\Omega$ is compact, every motion in $\Omega$ is compact. Therefore, if $\left(x^{*}, f^{*}\right) \in \Omega$, then the $\alpha$-limit set of the motion $\pi\left(x^{*}, f^{*}, t\right)$ is nonempty and lies in $\Omega \subset A(M)$. Since $M$ is a stable attractor, one has (see [2, Theorem 5]) $\Omega \subset M$. Therefore the positive limit set $L_{\left(x, f_{z}\right)}^{+}=\{0\}$, that is, $\phi\left(x, f_{\imath}, t\right) \rightarrow 0$ as $t \rightarrow \infty$. Q.E.D. 
REMARK. The author feels that one should be able to prove Theorem 6 without assuming that the null solution of $x^{\prime}=f(x, t)$ be uniformly stable. It may be necessary to assume that the null solutions of the limiting equations are uniformly asymptotically stable. If this is true, it would generalize a result of $L$. Markus [8, Theorem 2] for asymptotically autonomous equations.

D. Examples of limiting equations. Let us now consider some example of sets of limiting equations. We have already discussed asymptotically autonomous and the asymptotically periodic equations. Also, examples of asymptotically almost periodic equations appear in the literature. (See [11], [18].) We begin with the following example.

EXAmple B. Let $a, b$, and $c$ be real numbers and consider the second order equation

or more generally,

$$
a x^{\prime \prime}+b x^{\prime}+c x=\sin |t|^{1 / 2}
$$

$$
a x^{\prime \prime}+b x^{\prime}+c x=\sin \alpha(t)=g(t),
$$

where $\alpha$ is a diffeomorphism of $R^{+}$onto $R^{+}$and $\alpha^{\prime}(t) \rightarrow 0$ as $t \rightarrow \infty$. It is easily shown that the set of limiting equations for (9) is

$$
a x^{\prime \prime}+b x^{\prime}+c x=\mu \quad(-1 \leqq \mu \leqq 1)
$$

where $\mu$ is a constant. If the coefficients $a, b$, and $c$ are positive, then the solutions of (9) are uniformly asymptotically stable. If $\left\{\tau_{n}\right\}$ is a sequence with $\tau_{n} \rightarrow \infty$ and $g\left(\tau_{n}\right) \rightarrow \mu$, and if $\phi(t)$ is any solution of (9), then (by Theorems 2 and 5) one has $\phi\left(\tau_{n}\right) \rightarrow \mu / c$.

These facts are easily proved. We state them here to motivate the following generalization.

THEOREM 7. Let $f \in \mathfrak{S}\left(W \times R, R^{n}\right)$ and assume that $f$ is $\mathbf{C}^{1}$, that is, $f$ has continuous first partial derivatives. Assume that for each $\tau$ in $R$, there is a sequence $\left\{\tau_{n}\right\}$ with $\tau_{n} \rightarrow \infty$ and

$$
f\left(x, \tau_{n}\right) \rightarrow f(x, \tau) \quad(x \in W) .
$$

If $\partial f / \partial t \rightarrow 0$ as $t \rightarrow \infty$ (uniformly on compact sets in $W$ ), then every equation

$$
d x / d t=f(x, \tau) \quad(\tau \in R)
$$

is a limiting equation for $x^{\prime}=f(x, t)$. Moreover, the set of equations given in (12) is dense (with respect to the compact-open topology) in the set of limiting equations. Hence, every limiting equation for $x^{\prime}=f(x, t)$ is autonomous.

Proof. We shall first show that every equation of the form (12) is a limiting equation for $x^{\prime}=f(x, t)$. Indeed, let $\varepsilon>0$ be given and let $K=M \times I$ be a set in $W \times R$, where $M$ is compact in $W$ and $I$ is compact in $R$. We can assume, without any loss of generality, that $I$ is an interval with length $|I|$. Let $\tau \in R$ and choose 
$\left\{\tau_{n}\right\}$ in $R$ so that (11) is satisfied. We may assume that the convergence in (11) is uniform on $M$. Now choose $T>0$ so that

$$
|\partial f / \partial t(x, t)| \leqq \varepsilon \quad(x \in M, t \geqq T-|I|) .
$$

Choose $N$ so that $\tau_{n} \geqq T-|I|$ and

$$
\left|f\left(x, \tau_{n}\right)-f(x, \tau)\right| \leqq \varepsilon,
$$

for $n \geqq N$. It now follows from (13) and (14) that

$$
\begin{aligned}
\left|f\left(x, \tau_{n}+t\right)-f(x, \tau)\right| & \leqq\left|f\left(x, \tau_{n}+t\right)-f\left(x, \tau_{n}\right)\right|+\left|f\left(x, \tau_{n}\right)-f(x, \tau)\right| \\
& \leqq \varepsilon \cdot|I|+\varepsilon
\end{aligned}
$$

for $n \geqq N$ and $t \in I$. This proves that (12) is a limiting equation for $x^{\prime}=f(x, t)$.

Any other limiting equation for $x^{\prime}=f(x, t)$ is given by the limit (in the compactopen topology) of a sequence $\left\{f_{\tau_{n}}\right\}$ where $\tau_{n} \rightarrow \infty$. If $g \in \Omega_{f}^{*}$ gives rise to such an equation, then $g(x, t)=\lim f\left(x, \tau_{n}+t\right)$; that is $g$ lies in the closure of the set of equations (12). Q.E.D.

A stronger form of Theorem 7 is possible.

THEOREM 8. Let $f \in \mathfrak{S}\left(W \times R, R^{n}\right)$ and assume that $f=g+h$ where $g$ and $h$ are in $\mathfrak{\Im}\left(W \times R, R^{n}\right)$. Assume that $g_{t}$ is a positively compact motion. Furthermore, assume that for each $\tau$ in $R$ there is a sequence $\left\{\tau_{n}\right\}$ with

$$
\tau_{n} \rightarrow \infty \text { and } h\left(x, \tau_{n}\right) \rightarrow h(x, \tau) \quad(x \in W),
$$

and that $\partial h / \partial t \rightarrow 0$ as $t \rightarrow \infty$ (uniformly on compact sets in $W$ ). Then every equation of the form

$$
x^{\prime}=g^{*}(x, t)+h(x, \tau) \quad\left(\tau \in R, g^{*} \in \Omega_{g}^{*}\right)
$$

is a limiting equation of $x^{\prime}=f(x, t)$. Moreover, the set of all equations given in (15) is dense in the set of limiting equations for $f$.

The proof of this is similar to that of Theorem 7, so we shall omit the details. There are some immediate consequences of this result.

Corollary. Let $f, g$, and $h$ be given as in Theorem 8 .

(A) If $g$ is asymptotically autonomous, then (15) becomes

$$
x^{\prime}=g^{*}(x)+h(x, \tau) \quad(\tau \in R),
$$

where $\Omega_{g}^{*}=\left\{g^{*}\right\}$. In particular, every limiting equation for $x^{\prime}=g(x, t)$ is autonomous.

(B) If $g$ is asymptotically periodic, then (15) becomes

$$
x^{\prime}=g^{*}(x, \sigma+t)+h(x, \tau) \quad(\tau \in R, 0 \leqq \sigma<P),
$$

where $g^{*} \in \Omega_{g}^{*}$ has minimal period $P$. In particular, every limiting equation for $x^{\prime}=f(x, t)$ is periodic, of period $P$.

(C) If $g$ is asymptotically almost periodic, then every limiting equation for $x^{\prime}=f(x, t)$ is given by a Bohr almost periodic function. 
Proof. Parts (A) and (B) are obvious. Part (C) follows from Theorem 10 and Theorem 18 in [22]. Q.E.D.

E. Other topologies. The above results can be readily generalized to other topologies on the space of translates $\mathfrak{F}$ of a given function $f$ in $\mathfrak{C}\left(W \times R, R^{n}\right)$. The essential fact to note is that the definition of the "set of limiting equations" will now depend on this topology. In particular, if $f \in \mathfrak{C}\left(W \times R, R^{n}\right)$ and $\mathfrak{F}^{*}$ is any hull of $f$, then we define the set of limiting equations (with respect to $\mathfrak{F}^{*}$ ) of $x^{\prime}=f(x, t)$ by

$$
x^{\prime}=f^{*}(x, t) \quad\left(f^{*} \in \Omega_{f}^{*}\right),
$$

where $\Omega_{f}^{*}$ is the $\omega$-limit set of the motion $f_{t}$ in $\mathfrak{F}^{*}$. With this modification, all the results given above carry over with little change.

Let us note that many investigations of Bohr almost periodic differential equations can be viewed in terms of the Bohr topology on $\mathfrak{F}$ or $\mathfrak{F}_{b}^{*}$. In this case, a sequence $\left\{f_{\tau_{n}}\right\}$ in $\mathfrak{F}_{b}^{*}$ converges to a function $f^{*}$ in $\mathfrak{F}_{b}^{*}$ if and only if $\left\{f_{\tau_{n}}(x, t)\right\}$ converges to $f^{*}(x, t)$ uniformly on every set $M \times R$, where $M$ is compact in $W$. However, even in this case, one cannot expect to get a stronger conclusion in the analogue of Theorem 2. That is (using the notation of Theorem 2), the sequence of solutions $\phi\left(x, f, \tau_{n}+t\right)$ will not-in general-converge uniformly to $\phi\left(x^{*}, f^{*}, t\right)$.

4. Existence of almost periodic and periodic solutions. We now consider the following problems. Let $f \in \mathfrak{E}\left(W \times R, R^{n}\right)$ be a regular function. We ask then, under what (topological) conditions does the equation $x^{\prime}=f(x, t)$ have a Bohr almost periodic solution, or a periodic solution. This problem has been studied in various contexts by several authors. (See [4], [9], [12], [17], [18], [19], [21].) A related problem is to determine when the limiting equations for $x^{\prime}=f(x, t)$ have almost periodic, or periodic, solutions. We shall now study this and, as before, we assume that the space of translates $\mathfrak{F}$ is given with the compact-open topology.

A. Almost periodic solutions. We shall see that the following fact from topological dynamics will play a central role in this discussion.

THEOREM 9. Let $\pi$ be a local dynamical system on a metric space $X$ and let $\pi(p, t)$ be a positively compact motion in $X$. If $\pi(p, t)$ is uniformly positively Lyapunov-stable with respect to $\gamma^{+}(p) \cup \Omega_{p}$, then $\Omega_{p}$ is a.p. minimal.

A proof of this for dynamical systems can be found in [4]. However, that argument can easily be modified for local dynamical systems.

The definition of uniform stability appears in $[22, \S 2]$. However, it will be helpful to reformulate it here for the given local dynamical system $\pi$ on $W \times \mathfrak{F}_{\text {co. }}^{*}$.

Let $f \in \mathfrak{C}=\mathfrak{C}\left(W \times R, R^{n}\right)$ be a regular function and assume that the motion $\pi(x, f ; t)$, given by $(2)$, is defined for all $t \geqq 0$. Let $\rho$ be a basic metric on $\Subset$. The motion $\pi(x, f, t)$ is then uniformly positively Lyapunov-stable with respect to $D$ if for every $\varepsilon>0$ there is a $\delta>0$ such that

$$
d\left(\pi(\hat{x}, \hat{f} ; t), \pi\left(x^{*}, f^{*} ; t\right)\right)=\left|\phi(\hat{x}, \hat{f}, t)-\phi\left(x^{*}, f^{*}, t\right)\right|+\rho\left(\hat{f}_{t}, f_{t}^{*}\right)<\varepsilon,
$$


for all $t \geqq 0$, whenever

$$
(\hat{x}, \hat{f}) \in \gamma^{+}(x, f), \quad\left(x^{*}, f^{*}\right) \in D
$$

and $d\left((\hat{x}, \hat{f}),\left(x^{*}, f^{*}\right)\right)=\left|\hat{x}-x^{*}\right|+\rho\left(\hat{f}, f^{*}\right)<\delta$.

In the next theorem, and its corollaries, we seek necessary conditions that a given differential equation $x^{\prime}=f(x, t)$ has a uniformly positively Lyapunov-stable motion $\pi(x, f ; t)$. Because of Theorem 9 , we shall be interested in only positively compact motions.

THEOREM 10. Let $f \in \mathbb{E}\left(W \times R, R^{n}\right)$ be a regular function and let $\pi(x, f ; t)$ be a motion in $W \times \mathfrak{F}_{c o}^{*}$ that is positively compact and uniformly positively Lyapunovstable with respect to $\gamma^{+}(x, f) \cup \Omega_{(x, f)}$. Then the $\omega$-limit set $\Omega_{(x, f)}$ is a.p. minimal and the motion $f_{t}$ is positively compact. Hence, if $\left(x^{*}, f^{*}\right) \in \Omega_{(x, f)}$, then the solution $\phi\left(x^{*}, f^{*}, t\right)$ of $x^{\prime}=f^{*}(x, t)$ is Bohr almost periodic and the function $f^{*}(x, t)$ is Bohr almost periodic.

Proof. The fact that $\Omega_{(x, f)}$ is a.p. minimal follows from Theorem 9 . If $\pi\left(x^{*}, f^{*} ; t\right)$ is a motion in $\Omega_{(x, f)}$, then the projections

$$
P\left(\pi\left(x^{*}, f^{*} ; t\right)\right)=\phi\left(x^{*}, f^{*}, t\right) \text { and } Q\left(\pi\left(x^{*}, f^{*}, t\right)\right)=f_{t}^{*}
$$

into $W$ and $\mathfrak{F}_{\text {co }}^{*}$ are Bohr almost periodic. Q.E.D.

In the last theorem we showed that every limiting equation for $x^{\prime}=f(x, t)$ is Bohr almost periodic. One may then ask whether $f$ is asymptotically almost periodic. The answer is in the affirmative.

COROLlARY 1. Let f be given as in Theorem 10. If there exists a positively compact motion $\pi(x, f ; t)$ in $W \times \mathfrak{F}_{c o}^{*}$ that is uniformly positively Lyapunov-stable with respect to $\gamma^{+}(x, f) \cup \Omega_{(x, f)}$, then $f$ is asymptotically almost periodic.

Proof. We already observed that every function $f^{*}$ in $\Omega_{f}^{*}$ is Bohr almost periodic. It only remains to show that $\Omega_{f}^{*}$ is a minimal set. However, this is a direct consequence of the fact that $\Omega_{(x, f)}$ is a minimal set in $W \times \mathfrak{F}_{c o}^{*}$ (Theorem 10) and that the projection $Q$ of $W \times \mathfrak{F}_{\text {co }}^{*}$ onto $\mathfrak{F}_{c o}^{*}$ is continuous. Q.E.D.

One may ask whether the given equation $x^{\prime}=f(x, t)$ has a Bohr almost periodic solution. To this end we can prove the following:

CoROllary 2. Let $f$ be given as in Theorem 10 and assume that the motion $f_{t}$ is positively Poisson-stable, in the compact-open topology. Assume further that there is a positively compact motion $\pi(x, f ; t)$ in $W \times \mathfrak{F}_{c o}^{*}$ that is uniformly positively Lyapunov-stable with respect to $\gamma^{+}(x, f) \cup \Omega_{(x, f)}$. Then the equation $x^{\prime}=f(x, t)$ has a Bohr almost periodic solution.

Proof. By Theorem 10, the $\omega$-limit set $\Omega_{(x, f)}$ is a.p. minimal. Since the motion $f_{t}$ is Poisson-stable, there is a sequence $\left\{\tau_{n}\right\}, \tau_{n} \rightarrow \infty$, with $f_{\tau_{n}} \rightarrow f$. Furthermore, since the motion $\pi(x, f ; t)$ is positively compact, the solution $\phi(x, f, t)$ is positively compact. Therefore, the sequence $\left\{\phi\left(x, f, \tau_{n}\right)\right\}$ has a convergent subsequence, say 
that $\phi\left(x, f, \tau_{n}\right) \rightarrow \hat{x}$. Since $(\hat{x}, f) \in \Omega_{(x, f)}$, the solution $\phi(\hat{x}, f, t)$ of $x^{\prime}=f(x, t)$ is Bohr almost periodic by Theorem 10. Q.E.D.

The hypotheses of the last corollary deserve further comment. There exist regular functions $f$ in $\Subset\left(W \times R, R^{n}\right)$ that are not Bohr almost periodic, but have the following properties:

(i) the motion $f_{t}$ is positively compact (in the compact-open topology), and

(ii) the motion $f_{t}$ is positively Poisson-stable (in the compact-open topology).

(See L. Auslander and F. Hahn [1].) However, it is not possible that this type of function have a motion $\pi(x, f ; t)$ that is uniformly stable with respect to $\gamma^{+}(x, f) \cup \Omega_{(x, f)}$. This gives the next result.

COROLlary 3. Let $f \in \mathfrak{C}\left(W \times R, R^{n}\right)$ be a regular function where the motion $f_{t}$ is positively compact and positively Poisson-stable in the compact-open topology. A necessary condition that there be a positively compact motion $\pi(x, f ; t)\left(\right.$ in $\left.W \times \mathfrak{F}_{\mathrm{co}}^{*}\right)$ that is uniformly stable with respect to $\gamma^{+}(x, f) \cup \Omega_{(x, f)}$ is that f be a Bohr almost periodic function.

Proof. This follows from Corollary 2 and Theorem 10. Q.E.D.

The above results give necessary conditions (on $f$ ) that a positively compact motion $\pi(x, f ; t)$ be uniformly positively Lyapunov-stable, (with respect to $\left.\gamma^{+}(x, f) \cup \Omega_{(x, f)}\right)$. By also considering the corresponding solution $\phi(x, f, t)$ one can get both necessary and sufficient conditions. To do this we need to introduce a type of stability for solutions of $x^{\prime}=f(x, t)$ that is related to "total stability" or "stability under persistent disturbances." (See [5], [6], [10].)

Definition. Let $f \in \mathbb{C}\left(W \times R, R^{n}\right)$ be a regular function, let $\&$ be a subset of the hull $\mathfrak{F}_{\text {co }}^{*}$, and let $\rho$ be a basic metric on $\mathfrak{F}_{c o}^{*}$. We say that a solution $\phi(x, f, t)$ of $x^{\prime}=f(x, t)$ is stable under disturbances from is if for every $\varepsilon>0$ there is a $\delta>0$ such that

$$
|\phi(x, f, \tau+t)-\phi(y, g, t)| \leqq \varepsilon \quad(t \geqq 0)
$$

whenever $g \in \mathbb{S}$, and $|\phi(x, f, \tau)-y| \leqq \delta$ and $\rho\left(f_{\tau}, g\right) \leqq \delta$, for some $\tau \geqq 0$.

Before turning to the next theorem it will be convenient to reformulate the last type of stability as a comparison statement. It is easy to see that a solution $\phi(x, f, t)$ of $x^{\prime}=f(x, t)$ is stable under disturbances from \&s if and only if there is a continuous real-valued function $\alpha(r, s)$ defined for $0 \leqq r<a_{1}, 0 \leqq s<a_{2}$, such that $\alpha$ is increasing in each variable separately, $\alpha(0,0)=0$ and

$$
|\phi(x, f, \tau+t)-\phi(y, g, t)| \leqq \alpha\left(|\phi(x, f, \tau)-y|, \rho\left(f_{\tau}, f\right)\right)
$$

for all $t \geqq 0, \tau \geqq 0$, and $g \in$ Fs.

If the solution $\phi(x, f, t)$ of $x^{\prime}=f(x, t)$ is stable under disturbances from $\mathbb{B}$, then for every $\tau \geqq 0$ the solution

$$
\phi\left(\phi(x, f, \tau), f_{\tau}, t\right)=\phi(x, f, \tau+t)
$$

of $x^{\prime}=f_{\tau}(x, t)$ is stable under disturbances from $\mathfrak{S}$. Moreover, we can prove the following result. 
Lemma 6. Let $f \in \mathbb{E}\left(W \times R, R^{n}\right)$ be a regular function and let $\phi(x, f, t)$ be a positively compact solution of $x^{\prime}=f(x, t)$ that is stable under disturbances from $\mathbb{S}$. If $\left(x^{*}, f^{*}\right) \in \Omega_{(x, f)}$, then the compact solution $\phi\left(x^{*}, f^{*}, t\right)$ of the limiting equation $x^{\prime}=f^{*}(x, t)$ is stable under disturbances from (5). Moreover, if $\alpha$ satisfies (18) for $\phi(x, f, t)$, then the same $\alpha$ can be used for $\phi\left(x^{*}, f^{*}, t\right)$.

Proof. By the change of variables used in $\$ 3, \mathrm{C}$ we can assume that $\phi(x, f, t)=0, t \geqq 0$. In this case, inequality (18) becomes

$$
|\phi(y, g, t)| \leqq \alpha\left(|y|, \rho\left(f_{\imath}, g\right)\right)
$$

for all $t \geqq 0$ and all $g \in$ Ss. We now show that the null solution of every limiting equation is stable under disturbances from \&s.

Indeed, if $f^{*} \in \Omega_{f}^{*}$, then there is a sequence $\left\{f_{\tau_{n}}\right\}$ with $\tau_{n} \rightarrow \infty$ and $f_{\tau_{n}} \rightarrow f^{*}$. By continuity we get

$$
\rho\left(f_{\tau_{n}}, g\right) \rightarrow \rho\left(f^{*}, g\right), \quad \alpha\left(|y|, \rho\left(f_{\tau_{n}}, g\right)\right) \rightarrow \alpha\left(|y|, \rho\left(f^{*}, g\right)\right)
$$

and therefore

$$
|\phi(y, g, t)| \leqq \alpha\left(|y|, \rho\left(f^{*}, g\right)\right)
$$

for every $g \in(S)$ and every $f^{*} \in \Omega_{f}^{*}$. Q.E.D.

In our applications we shall be interested in the case where the set $\mathscr{S}$ mentioned above contains the set $\Omega *$.

We are now able to prove the following result which gives a sufficient condition that there exists a motion $\pi\left(x^{*}, f^{*}, t\right)$ that is positively compact and uniformly positively Lyapunov-stable with respect to $\gamma^{+}\left(x^{*}, f^{*}\right) \cup \Omega_{\left(x^{*}, f^{*}\right)}$.

THEOREM 11. Let $f \in \mathbb{E}\left(W \times R, R^{n}\right)$ be a regular function that is asymptotically almost periodic. Let $\phi(x, f, t)$ be a positively compact solution of $x^{\prime}=f(x, t)$ that is stable under disturbances from $\Omega_{f}^{*}$. Then every motion $\pi\left(x^{*}, f^{*}, t\right)$ in the $\omega$-limit set $\Omega_{(x, f)}$ is compact and uniformly positively Lyapunov stable with respect to $\Omega_{(x, f)}$. In particular, $\Omega_{(x, f)}$ contains an a.p. minimal set.

Proof. We choose $\alpha(r, s)$ so that (18) is satisfied. From Lemma 6 it follows that for every $g \in \mathbb{S}=\Omega_{f}^{*}$ one has

$$
\left|\phi\left(x^{*}, f^{*}, t\right)-\phi(y, g, t)\right| \leqq \alpha\left(\left|x^{*}-y\right|, \rho\left(f^{*}, g\right)\right) \quad(t \geqq 0)
$$

for every $f^{*} \in \Omega_{f}^{*}$, where $\left(x^{*}, f^{*}\right) \in \Omega_{(x, f)}$. In particular, (19) holds when $(y, g)$ are in $\Omega_{(x, f)}$ also. Let $\varepsilon>0$ be given. Since $\Omega_{f}^{*}$ is an a.p. minimal set, it is equicontinuous and there is a $\delta>0$ such that

$$
\rho\left(f_{t}^{*}, g_{t}\right) \leqq \varepsilon
$$

whenever $f^{*}, g$ are in $\Omega_{f}^{*}$ and $\rho\left(f^{*}, g\right) \leqq \delta$. Since $\alpha(r, s)$ is continuous, $\delta$ can also be chosen so that (19) implies that

$$
\left|\phi\left(x^{*}, f^{*}, t\right)-\phi(y, g, t)\right| \leqq \varepsilon \quad(t \geqq 0)
$$


whenever $\left(x^{*}, f^{*}\right) \in \Omega_{(x, f)},(y, g) \in \Omega_{(x, f)}$ and

$$
\left|x^{*}-y\right|+\rho\left(f^{*}, g\right) \leqq \delta .
$$

But this implies that the compact motion $\pi\left(x^{*}, f^{*}, t\right)$ is uniformly positively Lyapunov-stable with respect to $\Omega_{(x, f)}$. Q.E.D.

As a direct consequence of Theorems 10 and 11 we get the next result.

THEOREM 12. Let $f \in \mathbb{E}\left(W \times R, R^{n}\right)$ be a regular function that is asymptotically almost periodic. If there exists a positively compact solution $\phi(x, f, t)$ of

$$
x^{\prime}=f(x, t)
$$

that is stable under disturbances from $\Omega_{f}^{*}$, then every limiting equation of (20) has a Bohr almost periodic solution. If, in addition, $f$ itself is Bohr almost periodic, then (20) has a Bohr almost periodic solution.

REMARKS. 1. It should be noted that the second part of Theorem 12 has been discussed elsewhere by R. K. Miller [12] and G. Seifert [19]. Seifert's result, which is formulated in terms of the fundamental frequencies for the almost periodic differential equation, is equivalent to ours.

2. The concept of "stability under disturbances from \&s" is weaker than the concept of "total stability," or "stability under persistent disturbances." The latter concept has been discussed often in the literature and several sufficient conditions for this type of stability are known. We refer the reader to the papers [5], [6], [10].

B. Periodic solutions. In this section we shall be interested in (topological-dynamical) criteria for the existence of periodic solutions of differential equations. In a sense, this can be viewed as a generalization of the problems considered by Poincaré and Bendixson in their analysis of autonomous differential equations in the plane. Several authors [8], [9], [16], [21] have studied problems related to this. The problem is to assume that a given equation $x^{\prime}=f(x, t)$ has a positively compact solution $\phi(x, f, t)$ and to determine whether the given equation (or one of the limiting equations) has a periodic solution. For low order equations $\left(W \subset R^{2}\right.$, or $\left.W \subset R^{1}\right)$ one can get the desired conclusion, with very few additional hypotheses, by using either the Poincaré-Bendixson Theorem (cf. [8], [9], [16]) or a fixed-point theorem (cf. [9]). For the general problem, an asymptotic stability condition is needed for the desired conclusion (cf. [21]).

J. L. Massera [9] considered the problem of periodic solutions of periodic differential equations on $R^{n} \times R$. However, only for $n=1$ and $n=2$ was he able to state some (topological-dynamical) results on the existence problem mentioned above. These results can be generalized to asymptotically periodic equations.

THEOREM 13. Let $f \in \mathbb{C}(W \times R, R)$ be a regular function that is asymptotically periodic, where $W \subset R$. If $\phi(x, f, t)$ is a positively compact solution of $x^{\prime}=f(x, t)$, then the $\omega$-limit set $\Omega_{(x, f)}$ contains a periodic motion. In particular, every limiting equation of $x^{\prime}=f(x, t)$ has a periodic solution. 
Proof. Let $\left(x^{*}, f^{*}\right) \in \Omega_{(x, f)}$, where $\phi(x, f, t)$ is positively compact. Then, by Theorem 2, the solution $\phi\left(x^{*}, f^{*}, t\right)$ of

$$
x^{\prime}=f^{*}(x, t)
$$

is compact. Also, $f^{*}$ is periodic in $t$ since $f$ is asymptotically periodic. By a result of Massera [9], the solution $\phi\left(x^{*}, f^{*}, t\right)$ limits to a periodic solution of (21), and moreover, the periods are the same. Therefore, this periodic solution determines a periodic motion in $\Omega_{(x, f)}$. Q.E.D.

THEOREM 14. Let $f \in \mathfrak{C}\left(R^{2} \times R, R^{2}\right)$ be a regular function that is asymptotically periodic and let $f^{*} \in \Omega_{f}^{*}$. Assume that every solution $\phi\left(x^{*}, f^{*}, t\right)$ of the limiting equations $x^{\prime}=f^{*}(x, t)$ are defined for all $t \geqq 0$. If there is a positively compact solution $\phi(x, f, t)$ of $x^{\prime}=f(x, t)$, then every limit equation has a periodic solution, and the periods of the equation and the solution are the same.

The proof of this is similar to that of Theorem 13. It relies on Theorem 2 and a result of J. L. Massera [9] for periodic equations on $R^{2} \times R$. We shall omit the details. However, one should note that the periodic motions on $W \times \mathfrak{F}_{c o}^{*}$ may not be in the $\omega$-limit set $\Omega_{(x, f)}$, as was the case in the last theorem.

For higher order equations, the following theorem is needed.

THEOREM 15. Let $\pi$ be a local dynamical system on a metric space $X$. If $\pi(p, t)$ is a positively compact motion that is asymptotically stable with respect to $\gamma^{+}(p) \cup \Omega_{p}$, then $\Omega_{p}$ is a minimal set which contains a single periodic trajectory.

The definition of asymptotic stability appears in [22, \$2] and the proof of this theorem-for dynamical systems-appears in [21]. The proof can be easily modified for local dynamical systems.

Let us reformulate the concept of asymptotic stability for the given local dynamical system $\pi$ on $W \times \mathfrak{F}_{\text {co. }}^{*}$. Let $f \in \mathfrak{C}=\mathfrak{C}\left(W \times R, R^{n}\right)$ be a regular function and assume that the motion $\pi(x, f ; t)$ is defined for all $t \geqq 0$. Let $\rho$ be a basic metric on $\mathbb{E}$. The motion $\pi(x, f ; t)$ is asymptotically stable with respect to $D$ if

(i) $\pi(x, f ; t)$ is uniformly positively Lyapunov-stable with respect to $D$ (see above), and

(ii) there is an $\eta_{0}>0$ with the property that for every $\left(x^{*}, f^{*}\right)$ in $D$ with

$$
\left|x^{*}-\phi(x, f, \sigma)\right|+\rho\left(f^{*}, f_{\sigma}\right)<\eta_{0} \quad \text { (for some } \sigma \geqq 0 \text { ), }
$$

there is a $\tau$ in $R$ such that

$$
\left|\phi\left(x^{*}, f^{*}, t\right)-\phi(x, f, \tau+t)\right|+\rho\left(f_{t}^{*}, f_{\tau+t}\right) \rightarrow 0 \quad(\text { as } t \rightarrow \infty) .
$$

We now seek necessary conditions that the given differential equation $x^{\prime}=f(x, t)$ has an asymptotically stable motion $\pi(x, f ; t)$. Because of Theorem 15 , we shall be interested in only positively compact motions. Since asymptotic stability implies uniform positive Lyapunov-stability, some necessary conditions appear in Theorem 10 and its corollaries. 
THEOREM 16. Let $f \in \mathbb{C}\left(W \times R, R^{n}\right)$ be a regular function and let $\pi(x, f ; t)$ be a motion in $W \times \mathfrak{F}_{\mathrm{co}}^{*}$ that is positively compact and asymptotically stable with respect to $\gamma^{+}(x, f) \cup \Omega_{(x, f)}$. Then $f$ is asymptotically periodic and the $\omega$-limit set $\Omega_{(x, f)}$ consists of a single periodic trajectory. Hence, if $\left(x^{*}, f^{*}\right) \in \Omega_{(x, f)}$, then $f^{*}$ is periodic and the solution $\phi\left(x^{*}, f^{*}, t\right)$ is periodic; furthermore, the periods are commensurable.

Proof. The argument of Theorem 10, together with Theorem 15, gives the desired result. Q.E.D.

The question of sufficient conditions that a motion $\pi(x, f ; t)$ be asymptotically stable seems to be harder than the corresponding question for uniform stability discussed in Theorem 11. The advantage of Theorem 11 is that the concept of "stability under disturbances from $(5)$ " is related to standard type of stability (viz. stability under persistent disturbances) which is fairly well understood. We are able to give a sufficient condition in terms of uniform asymptotic stability, but a much weaker condition is certainly possible.

THEOREM 17. Let $f \in \mathfrak{C}\left(W \times R, R^{n}\right)$ be a regular function that is asymptotically periodic. If $\phi(x, f, t)$ is a positively compact solution of $x^{\prime}=f(x, t)$ that is uniformly asymptotically stable, then $\Omega_{(x, f)}$ is periodic minimal. In particular, every limiting equation for $x^{\prime}=f(x, t)$ has a periodic solution.

Proof. It is easy to show that every limiting equation has a periodic solution. We proceed as follows. If $\phi(x, f, t)$ is a positively compact solution of $x^{\prime}=f(x, t)$ that is uniformly asymptotically stable, then (by Theorem 5 ) every limiting equation has a compact solution that is uniformly asymptotically stable. Since the limiting equations are periodic, it follows from [21, Theorem 4] that every limiting equation has a periodic solution.

The conclusion that $\Omega_{(x, f)}$ is periodic minimal is stronger than the mere existence of periodic solutions of the limiting equations. However, this follows from [21, Corollary to Theorem 5]. More precisely, if $\left(x^{*}, f^{*}\right) \in \Omega_{(x, f)}$, then $\phi\left(x^{*}, f^{*}, t\right)$ is a compact, uniformly asymptotically stable solution of $x^{\prime}=f^{*}(x, t)$. Furthermore, by the asymptotic stability, there is an $\eta_{0}>0$ such that if $\left|x-\phi\left(x^{*}, f^{*}, \tau\right)\right|<\eta_{0}$ for some $\tau$ in $R$, then

$$
\left|\phi\left(x, f^{*}, t\right)-\phi\left(x^{*}, f^{*}, \tau+t\right)\right| \rightarrow 0 \quad(\text { as } t \rightarrow \infty) .
$$

Hence, the set

$$
\mathrm{Cl} \gamma\left(x^{*}, f^{*}\right)=\mathrm{Cl}\left\{\left(\phi\left(x^{*}, f^{*}, t\right), f_{t}^{*}\right): t \in R\right\}
$$

is contained in the region of attraction of $\phi\left(x^{*}, f^{*}, t\right)$ (see [21] for definitions) and $\pi\left(x^{*}, f^{*}, t\right)$ is periodic. Q.E.D.

5. Generalizations. In conclusion, some comments can be made on possible areas of generalization. The first observation is a minor point. Although we assumed that the given differential equation $x^{\prime}=f(x, t)$ was defined on $W \times R$, it is possible to consider equations defined on $W \times R^{+}$. The natural way to do this 
is to modify everything and discuss only positive semitrajectories, positive invariance, positive recurrence, etc. Another way to treat these equations is to extend the given equation to all of $W \times R$ and employ the above results.

One may ask whether the conditions for admissibility (cf. [22, §3]) of the given equation can be weakened. It appears that both conditions can be weakened somewhat. We shall discuss each condition separately.

A. Discontinuous equations. If one wishes to relax the assumption that the given differential equation $x^{\prime}=f(x, t)$ be continuous, it seems natural to impose a Carathéodory-type hypothesis. That is, assume that

(i) $f$ is measurable,

(ii) for each $t, f(x, t)$ is continuous in $x$, and

(iii) for each compact set $K \subset W$, there is an integrable function $m(t)$ such that $|f(x, t)| \leqq m(t)$, for all $x$ in $K$ and $t$ in $R$.

The author is unable to give any specific results for this more general case. The problem with this approach seems to be in placing an appropriate topology on the spaces of translates $\mathfrak{F}$ so that the mapping $\pi$, defined by (2), defines a local dynamical system on $W \times \mathfrak{F}$. It would appear that a topology could be generated by means of an integrability condition similar to that used by Z. Opial [16].

B. Nonuniqueness. In the paper on asymptotically autonomous equations, L. Markus [8] does not assume that the limiting equations have unique solutions. Also, R. K. Miller [11] does not use a uniqueness hypothesis in his discussion of perturbed almost periodic equations. Needless to say, many of our conclusions can be generalized to this case. However, our viewpoint seems to require the uniqueness hypothesis.

It is interesting to ask whether uniqueness of solutions is critical. Some authors (Barbašin [3] and Minkevič [13]) have attempted to develop a theory of topological dynamics for autonomous differential equations $x^{\prime}=f(x)$, where $f$ is (only) continuous. However, as pointed out in [20, p. 385], their assumptions of continuity were too restrictive to apply to any known differential equation that does not have unique solutions.

The author [20] has shown that the solutions of $x^{\prime}=f(x)$ do satisfy a continuity condition, when $f$ is continuous. It would be interesting to know whether a topological-dynamical theory could be developed for the autonomous differential equation that lacks uniqueness and, further, whether this theory can be extended, in the spirit described in these papers, to nonautonomous differential equations.

Added in proof. The theory of topological dynamics can be used to study the solutions of discontinuous differential equations that satisfy a Carathéodory hypothesis. This extension of the present work is discussed in a forthcoming paper by R. K. Miller and the author, on the dynamics of Volterra integral equations. 


\section{REFERENCES}

1. L. Auslander and F. Hahn, Real functions coming from flows on compact spaces and concepts of almost periodicity, Trans. Amer. Math. Soc. 106 (1963), 415-426.

2. J. Auslander, N. P. Bhatia and P. Seibert, Attractors in dynamical systems, Bol. Soc. Mat. Mexicana (1964), 55-66.

3. E. A. Barbašin, On the theory of general dynamical systems, Učen. Zap. Moskov. Gos. Univ. 135 Matematika 2 (1948), 110-133. (Russian)

4. L. G. Deysach and G. R. Sell, On the existence of almost periodic motions, Michigan Math. J. 12 (1965), 87-95.

5. W. Hahn, On the general concept of stability and Lyapunov's direct method, MRC Tech. Rep. 485, Univ. of Wisconsin, Madison, 1964.

6. N. N. Krasovskii, Stability of motion, Stanford Univ. Press, Palo Alto, Calif., 1963.

7. J. P. LaSalle, Asymptotic stability criteria, Proc. Sympos. Appl. Math. Vol. 13, pp. 299-307, Amer. Math. Soc., Providence, R. I., 1962.

8. L. Markus, Asymptotically autonomous differential systems, Contributions to Nonlinear Oscillations, Vol. 3, Princeton Univ. Press, Princeton, N. J., 1956.

9. J. L. Massera, The existence of periodic solutions of systems of differential equations, Duke Math. J. 17 (1950), 457-475.

10. - Contributions to stability theory, Ann. of Math. (2) 64 (1956), 182-206.

11. R. K. Miller, Asymptotic behavior of solutions of nonlinear differential equations, Trans. Amer. Math. Soc. 115 (1965), 400-416.

12. - Almost periodic differential equations as dynamical systems with applications to the existence of a.p. solutions, J. Differential Equations 1 (1965), 337-345.

13. M. I. Minkevič, Theory of integral funnels in dynamical systems, Učen. Zap. Moskov. Gos. Univ. 135 Matematika 2 (1948), 134-151. (Russian)

14. V. V. Nemyckii and V. V. Stepanov, Qualitative theory of differential equations, Princeton Univ. Press, Princeton, N. J., 1960.

15. V. V. Nemyckii,, Topological problems of the theory of dynamical systems, Uspehi Mat. Nauk 4 (1949), 91-153; English transl., Amer. Math. Soc. Transl. (1) 5 (1962), 414-497.

16. Z. Opial, Sur la dépendance des solutions d'un système d'équations différentielles de leurs seconds membres. Applications aux systèmes presque autonomous, Ann. Polon. Math. 8 (1960), 75-89.

17. C. R. Putnam, Unilateral stability and almost periodicity, J. Math. Mech. 9 (1960), 915-917.

18. G. Seifert, Stability conditions for the existence of almost-periodic solutions of almostperiodic systems, J. Math. Anal. Appl. 10 (1965), 409-418.

19. —_, Almost periodic solutions for almost periodic systems of ordinary differential equations, J. Differential Equations 2 (1966), 305-319.

20. G. R. Sell, On the fundamental theory of ordinary differential equations, J. Differential Equations 1 (1965), 370-392.

21. —_, Periodic solutions and asymptotic stability, J. Differential Equations 2 (1966), 143-157.

22. - Nonautonomous differential equations and topological dynamics. I. The basic theory, Trans. Amer. Math. Soc. 127 (1967), 241-262.

UNIVERSITY OF MINNESOTA, MinNeAPOLIS, MinNeSOTA 